\title{
Elimination de la rougeole: le but est à portée de main
}

\author{
Virginie Masserey Spicher \\ Dr med., cheffe de la section vaccinations et mesures de contrôle, Office fédéral de la santé publique
}

L’année 2015 est décisive pour l'élimination de la rougeole en Suisse, et tout le monde peut contribuer à l'atteinte de cet objectif. Dans le cadre de la Stratégie nationale d'élimination de la rougeole 2011-2015, qui bénéficie d'un large soutien, la Confédération, les cantons et les associations professionnelles ont déjà pris plusieurs mesures - avec des résultats encourageants.

\section{Les principaux objectifs de la stratégie}

1. parvenir, dans chaque nouvelle cohorte de naissances, à un taux d'au moins $95 \%$ des enfants de 2 ans protégés contre la rougeole avec deux doses;

2. combler, d'ici fin 2015 , les lacunes de vaccination chez les personnes nées après 1963;

3. maîtriser le plus rapidement possible les flambées de rougeole, par la mise en œuvre de mesures uniformisées dans tous les cantons.

\section{Les principales mesures}

Le Comité pour une Suisse sans rougeole, actif au niveau national avec quinze personnalités relevant de différents domaines - santé, sport, UNICEF, protection des consommateurs et politique - s'engage, tant sur le plan politique qu'auprès du grand public, en faveur d'un large soutien à l'élimination de la rougeole.

On s'adresse aux parents par différents canaux, en mettant l'accent sur l'importance de faire vacciner leurs enfants dans les temps et sur les conséquences de l'absence de vaccination: en cas de flambée de rougeole, notamment, ceux qui ne sont pas vaccinés peuvent

\section{Recommandations de vaccination}

- L'Office fédéral de la santé publique (OFSP) recommande comme vaccination de base pour tous les enfants la vaccination combinée contre la rougeole, les oreillons et la rubéole (ROR): la première dose à l'âge de 12 mois, et la seconde entre 15 et 24 mois.

Une vaccination de rattrapage (ROR) est possible à tout âge; elle est recommandée en particulier aux personnes nées après 1963 qui n'ont pas reçu deux doses de vaccin et qui n'ont pas eu la maladie.

- Pour les nourrissons qui présentent un risque élevé de contracter la rougeole (prématurés, enfants en crèche ou chez une maman de jour) et pendant une épidémie, la première dose ROR est recommandée à 9 mois et la seconde entre 12 et 15 mois. En cas de contact direct avec une personne malade, une première dose est recommandée dès l'âge de 6 mois.

- L'intervalle minimum entre deux doses est d'un mois. être temporairement exclus de la crèche ou de l'école. Pour cela, les autorités sanitaires cantonales envoient, par ex., des lettres personnelles aux parents, et les crèches et les écoles leur donnent des informations sur la vaccination au moment de l'inscription. Les pédiatres, quand ils reçoivent des parents de jeunes enfants, conviennent d'emblée d'un rendez-vous à la date recommandée pour la vaccination ROR ou les y invitent activement à l'âge dû.

Le carnet de vaccination électronique est préconisé. On peut le créer soi-même via une "app», sur www. mesvaccins.ch, ou se faire aider par un-e professionnel-le de la santé, idéalement, pour les nourrissons, dès les premières vaccinations chez le pédiatre. Le carnet inclut un système de rappel automatique, signalant les vaccinations qui manquent. Il est protégé par un mot de passe, mais est consultable à tout moment, on ne peut plus le perdre, et on peut l'imprimer. Une fois validé par un médecin ou un pharmacien, il est tout aussi valable qu'un carnet de vaccination sur papier.

Les jeunes et les adultes jusqu'à 50 ans sont particulièrement concernés par la vaccination de rattrapage jusqu'à deux doses, car ils ignorent souvent qu'ils ne sont pas ou pas suffisamment protégés. C'est pour cela que la Confédération et les cantons mènent ensemble, depuis 2013, la campagne nationale «Stop rougeole» (www.stoprougeole.ch). Elle vise principalement à inciter les jeunes et les adultes à contrôler leur statut vaccinal et à combler le plus vite possible les éventuelles lacunes au moyen d'une vaccination de rattrapage. Celle-ci est exemptée de franchise jusqu'à fin 2015 et, dans de nombreux cantons, des actions encouragent les gens à se faire vacciner.

Les médecins contrôlent systématiquement les carnets de vaccination et administrent les vaccinations de rattrapage nécessaires. Une extrapolation effectuée sur la base d'une enquête réalisée auprès des médecins de premier recours, dans le cadre du système de déclaration Sentinella, indique pour 2014, 33500 vaccinations de rattrapage chez des personnes de 2 à 50 ans. En dix mois, les répondants ont effectué en moyenne 4,4 vaccinations de rattrapage (généralistes et internistes: 3,5 doses, pédiatres: 9,1 doses). Un interniste en a même administré 109 et un pédiatre 107 ! 
Comment les professionnel-le-s de la santé peuvent-ils contribuer à éliminer la rougeole en Suisse?

1. en parlant de la rougeole: grâce à la vaccination, la maladie est devenue si rare qu'on en a presque oublié les manifestations les plus graves. Les professionnel-le-s de la santé doivent donc prendre au sérieux la crainte des effets secondaires des vaccins qu'ont certaines personnes, prendre au sérieux les doutes et, par des informations objectives, renforcer la confiance dans les recommandations relatives à la vaccination.

2. en prévenant la rougeole: les médecins peuvent tirer parti de tout contact avec des patient-e-s (par ex. aux urgences, avant un voyage à l'étranger ou lors d'un contrôle gynécologique) pour vérifier dans leur carnet de vaccination, le statut immunitaire en regard de la rougeole, de toute personne née après 1963 et pour la vacciner immédiatement ou lors du prochain rendez-vous. Par ailleurs, tous les professionnel-le-s, qu'ils exercent dans le domaine de la santé ou dans l'accueil des nourrissons, devraient être eux-mêmes vaccinés.

3. en pensant à la rougeole: tout cas suspect (triade: 1. fièvre, 2. exanthème maculo-papuleux, et 3. toux, rhinite ou conjonctivite) doit être aussitôt déclaré au service du médecin cantonal et confirmé (ou infirmé) par des analyses de laboratoire spécifiques à la rougeole.

Les «Directives de lutte contre la rougeole et les flambées de rougeole», publiées en 2013, posent le cadre permettant à tous les cantons de maîtriser la maladie rapidement et efficacement.

\section{Situation actuelle}

La couverture vaccinale a continué d'augmenter dans pratiquement tous les cantons et toutes les classes d'âge. Dans les 8 cantons ayant participé à l'enquête en 2014, 87\% des enfants de 2 ans étaient déjà protégés par deux doses de vaccin contre la rougeole. Un point particulièrement réjouissant est qu'il y a eu de nets progrès dans les cantons qui se situaient au-dessous de la moyenne nationale: par ex., la couverture est passée de 50 à $85 \%$ dans celui d'Appenzell Rhodes-Intérieures, de 76 à $82 \%$ dans celui de Schwyz et de 82 à $87 \%$ dans celui de Lucerne. Genève, Vaud et Fribourg ont déjà atteint les $95 \%$ visés chez les enfants de 8 ans. Genève est en outre le premier canton dans lequel le but visé de 95\% des enfants de 2 ans protégés par deux doses a été atteint. Pour que la rougeole soit considérée comme éliminée, il faut que 95\% de la population soit immune contre la maladie et qu'il n'y ait pas plus d'un cas de rougeole par million d'habitants et par an.

Le nombre de cas de rougeole confirmés a reculé en Suisse ces dernières années, passant de 664 en 2011, plus de 65 en 2012 et 176 en 2013 à 23 seulement en 2014, ce qui, pour cette dernière année, correspond à une incidence de 2,8 cas par million d'habitants. Ce critère d'élimination n'est donc pas encore tout à fait atteint. La maladie touche principalement des adolescents et des adultes sans protection vaccinale suffisante: l'âge moyen des malades de 2012 à 2014 était de 15 ans, et cette tendance se maintient en 2015. Dans la région Europe de l'OMS, $50 \%$ des pays sont actuellement exempts de rougeole. Mais plus de 16000 cas ont été recensés en 2014, avec des flambées en Italie, en Slovénie, en Bosnie et en Allemagne. Un enfant de 18 mois non vacciné est mort de la rougeole en février 2015 à Berlin. Le virus est exporté dans des pays exempts de rougeole par les voyageurs: en 2014, des flambées ont été observées au Brésil et, début 2015, aux Etats-Unis, où plus d'une centaine d'enfants et d'adultes la plupart non vaccinés ont contracté la maladie à partir d'un cas survenu à Disneyland.

L'objectif d'une Suisse sans rougeole est à portée de main. Mais des flambées de la maladie sont encore possibles à tout moment. Autrement dit, il vaut la peine de continuer à agir. La vaccination est un investissement pour la santé simple, sûr et efficace qui, de plus, entraîne des économies pour tous.

Le vendredi 6 novembre aura lieu la journée nationale Stop rougeole (le même jour que la journée de vaccination contre la grippe). La vaccination contre la rougeole est exemptée de la franchise jusqu'à fin 2015 pour tous ceux qui souhaitent se protéger de la maladie. Il est donc particulièrement intéressant de combler maintenant ses lacunes vaccinales.

\section{Pour de plus amples informations}

- www.stoprougeole.ch: site qui présente des informations et des illustrations sur la rougeole, le test de risque en ligne, la campagne (avec la possibilité de commander du matériel) ainsi que les actions menées dans les cantons.

- www.bag.admin.ch/rougeole: site de l'OFSP présentant les chiffres les plus récents, de nombreuses informations sur la maladie, la vaccination et la stratégie, ainsi que de la documentation destinée à la population et aux professionnel-le-s de la santé.

- www.mesvaccins.ch: site permettant de créer gratuitement son carnet de vaccination électronique: protégé par un mot de passe, celui-ci rappelle automatiquement les dates des vaccinations - et on ne le perd jamais! 